\title{
Kokospalme und Brotfruchtbaum: Siedlungs-Vorstellungen der Sawos und Kwanga, Sepik-Gebiet, Papua-Neuguinea
}

\section{Einleitung}

Die gedanklichen Beziehungen von Menschen zu ihrer gebauten Umgebung, zu ihrer Siedlung, am Beispiel von zwei Kulturen des Sepik-Gebietes in Papua-Neuguinea dargestellt, bilden das Thema der folgenden Ausführungen. Sie sollen einen Beitrag für jene Untersuchungen bieten, die man unter dem Titel «man-environment studies» zusammenfassen kann (1). Die gedanklichen Verbindungen von Menschen zu ihrer Siedlung werden an folgenden Aspekten dargelegt: Zuerst wird der Frage nachgegangen, wie sich die Siedlung in die räumliche Orientierung einfügt, welche Verbindungen zwischen der Siedlung und den mythischen Berichten über die Weltentstehung vorhanden sind und welche Folgerungen daraus für die gegenwärtige Lage des Dorfes gezogen werden. Daraufhin lege ich dar, welche Merkmale für eine Siedlungsgründung angeführt werden. Bei der Betrachtung der Siedlungsform tritt besonders der Tanzund Kultplatz in Erscheinung. SCHMITZ (1955) hat in einer vergleichenden Untersuchung die Tanz- und Kultplätze in Melanesien behandelt. Hier soll weniger das äußere Bild des Platzes oder seine Einordnung in den Dorfgrundriß betrachtet, sondern vielmehr den mit dem Kultplatz verbundenen Äußerungen der Einheimischen nachgegangen werden. SCHMITZ (1955: 8) schreibt: «Der „Tanz- und Kultplatz” repräsentiert sich zwar in einer materiellen Erscheinung, aber erst, was auf inm geschieht und welche Rolle er in der Vorstellung seiner Benutzer spielt, verleiht ihm Sinn und Bedeutung." Besonders am Beispiel der Sawos wird die zentrale Stellung des Kultplatzes deutlich werden. BÜHLER (1960) zeigt anhand von Beispielen aus Ostindonesien und Melanesien, wie Kultplätze zum Zentrum einer Siedlung werden können; eines seiner Beispiele bezieht sich auf Dörfer des Mittelsepik. BÜHLER (1960: 203) sagt, «daß die Bedeutung der religiös kultisch wichtigen Siedlungsteile stark überwiegt, daß sie oft die gesamte Siedlungsform bedingt. Kultanlagen scheinen sich hier als Siedlungskerne und damit für die Siedlungsform als primär bestimmend zu erweisen». Als vierten Aspekt der gedanklichen Beziehungen der Menschen zu ihrer Siedlung führe ich einzelne symbolische Äußerungen an, den letzten Punkt der Betrachtung bilden rituelle Handlungen, die sich auf das Dorf beziehen.

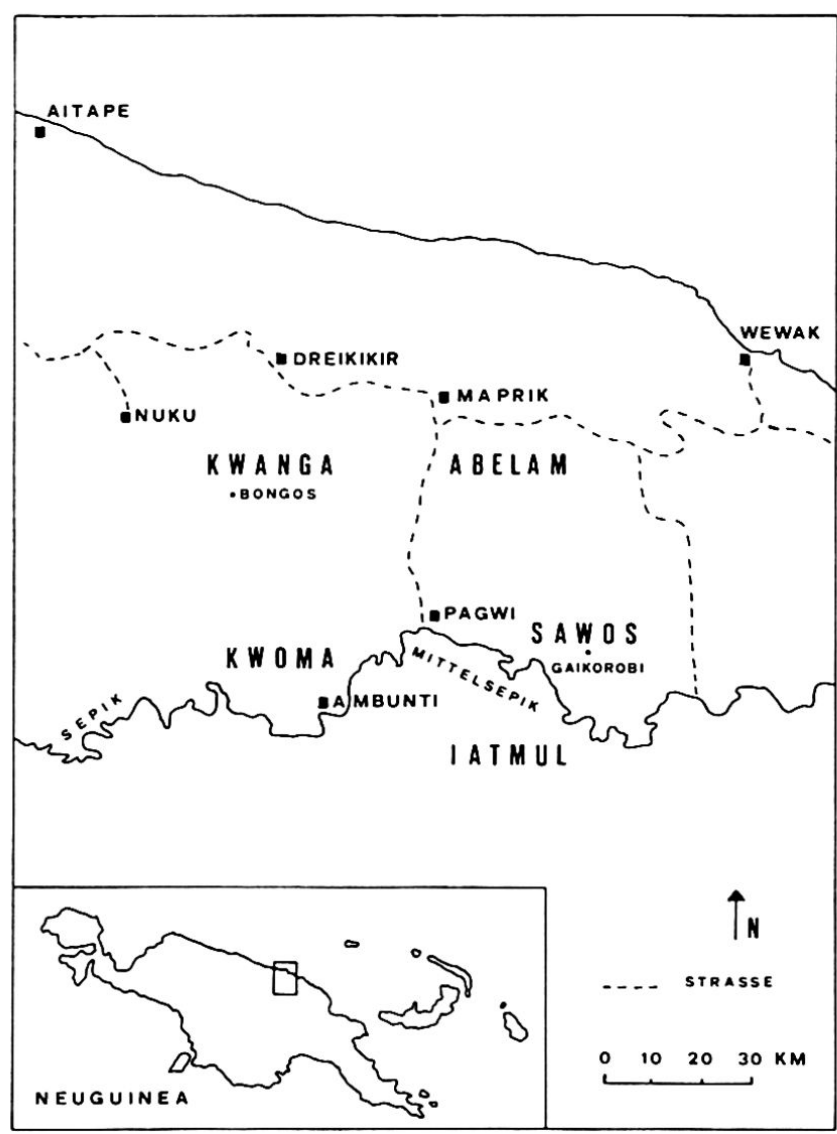

Karte des Mittelsepik-Gebietes und der Nordküste

\section{Die Sawos}

Die Sawos (2) mit einer Bevölkerungszahl von etwa 8000 Menschen leben in der östlichen Sepik-Provinz von Papua-Neuguinea. In ihrem Gebiet befinden sich reiche Sagopalmenbestände, Sümpfe, tropische Regenwälder und Grasländer. Das alljährliche Hochwasser des Sepik erreicht nur die südlichen SawosGebiete. Wie die Flußbewohner, die Iatmul, leben auch die Sawos in Pfahlbauten. Ihr Wirtschaftssystem basiert auf der Gewinnung von Sagostärke und auf dem Fischfang, ferner werden zusätzlich Fische von den Iatmul im Tausch gegen Sago eingehandelt. Man baut Yams, Taro, Bananen, Blattgemüse, Süßkartoffel

Dr. Markus Schindlbeck, Ethnologisches Seminar Münsterplatz 19, 4051 Basel 
und Mais an. Vielfältige Sammeltätigkeiten und Jagd bilden weitere Tätigkeiten der Sawos zur Nahrungsgewinnung. Die Einwohnerzahl der Sawos-Dörfer variiert zwischen ca. 100 und 400 Bewohnern pro Dorf. Die grundlegenden Verwandtschaftsgruppen sind patrilineare exogame Klane, die in eine Hälftenordnung gegliedert sind. Der Kontakt mit der europäischen Zivilisation begann zur Zeit des Ersten Weltkrieges.

\section{Die kosmologische Einordnung}

Die Dörfer der Sawos befinden sich nördlich des Mittelsepiks innerhalb einer weiten Alluvialebene, die im Norden und Süden von Gebirgen eingerahmt wird. Der Sepik-Strom fließt von Westen nach Osten dem Meere zu. In den Mythen und kosmologischen Erklärungen der Sawos finden wir eine Vier-Teilung vor, den vier Himmelsrichtungen entsprechend. Nach der Entstehung der Erde aus dem Urwasser heraus wurden die Landflächen und die Menschen verteilt. Die Sawos haben die Vorstellung von einem kosmischen Krokodil, das mit seinem Kopf nach Westen gerichtet ist. Der Rachen dieses Krokodiles ist in der Urzeit geöffnet worden. Der Oberkiefer bildet die Sonnenplattform oder auch eine Art Wolkenbett, der Unterkiefer stellt den Erdboden dar. Alle Menschen befinden sich im Inneren dieses Krokodilrachens. Die Kiefer werden von Pfosten auseinandergehalten. In einer anderen mythologischen Überlieferung spricht man von einer Miesmuschel. Männliche Urahnen zogen deren Teile in der Urzeit auseinander und setzten in den vier Himmelsrichtungen Pfosten in die Erde. Die Namen dieser vier Weltenpfosten sind heute noch von Bedeutung, wenn gegen ein fremdes Dorf die Ahnen zur Unterstützung angerufen werden. Das Dorf der Sawos steht somit innerhalb eines kosmischen Hauses oder auch Krokodiles darinnen. Das Dorf allein wird aber auch als ein Haus betrachtet, wobei die einzelnen Pfosten des Hauses die Repräsentanten wichtiger Klanvorfahren bilden. Diese Klanvorfahren werden als die Gründer des Dorfes bezeichnet. Sie tragen wie Pfosten und Balken ein Haus, das von ihren Nachfahren bewohnt wird. Wenn ein Balken oder Pfosten dieses Hauses gebrochen ist, d. h. wenn innerhalb der Dorfgemeinschaft ein Klan seine Aufgaben oder auch seine Stellung nicht mehr behalten kann, so droht dem gesamten Dorf Gefahr, besonders früher in kriegerischen Auseinandersetzungen mit Feinddörfern.

\section{Die Dorfgründung}

Die Darstellung des Dorfes als eines Hauses wird deutlicher, wenn wir die einheimischen Aussagen über die Gründung einer Siedlung betrachten: man vergleicht den Vorgang mit einem Hausbau. Dazu rodet man ein Waldstück, entfacht ein Feuer und hebt Pfostenlöcher aus. Das Setzen der Pfosten vergleicht man mit dem Bestatten der ersten Toten im Dorf. Die ersten Toten in einem Dorf, die im Dorfboden begraben werden, haben eine besondere Stellung. Sie sind die Gründer des Dorfes, auf ihrem Rücken, auf ihren Gebeinen spielt sich das Leben ihrer Nachfahren ab. Wenn man diese Siedlungsstelle wieder verläßt, sind es nach einheimischer Vorstellung diese Toten, die dafür sorgen, daß in einer neuen Siedlung Menschen zuerst sterben müssen; sie werden hier als todbringende Ahnen betrachtet. Dieses Erwidern des Todes der ersten Siedler bezieht sich nicht auf kleinere Siedlungen im Waldgebiet außerhalb des Dorfes, auf vereinzelt stehende Waldhäuser oder Weiler, sondern nur auf Siedlungen, die ein eigenes Männerhaus und einen eigenen Namen haben.

In diesem Zusammenhang ist ein Gedankengang wichtig, der oft in Argumentationen über Anrechte auf Bodennutzung auftritt. Dieser besagt, daß ein Anrecht auf ein Stück Waldgebiet usw., das nicht von den eigenen Klanvorfahren übernommen wurde, erst dadurch besteht, daß von der eigenen Gruppe Menschen sterben mußten, daß auf diese Weise die eigenen Toten in der Erde begraben sind und man von da an auf den Gebeinen der eigenen Toten geht. Für jedes Waldstück oder Palmenareal muß man so mit dem Leben eines Menschen der eigenen Gruppe bezahlen.

Bei der Gründung einer Siedlung wird aber nicht nur von Pfosten gesprochen, die wie beim Hausbau in die Erde gesetzt werden, sondern auch von einer Schlange, einem Rotang, einem Hüllblatt einer Palmenblüte, einer Sagobackschale und einer Herdschale aus Ton. Jeder Klanverband besitzt Namen, die sich auf diese Objekte beziehen, die man im zentralen Kultplatz des Dorfes Gaikorobi, im wompunau von Kosimbi, lokalisiert. Hierbei konnte nicht geklärt werden, inwiefern tatsächlich Objekte in die Erde hineingelegt werden, da die Ansichten der einheimischen Gewährsmänner darüber auseinandergingen. Wenn wir also auch nicht sagen können, daß tatsächlich eine Herdschale bei einer Dorfgründung auf dem Tanzplatz eingegraben wird, so besteht jedoch eindeutig die Vorstellung darüber, daß diese genannten Objekte eng mit dem Dorf als Einheit verknüpft sind. Das Auffallende ist, daß sich diese Aussagen in Gaikorobi immer auf einen ganz bestimmten Tanzoder Kultplatz beziehen, der nach einem früheren, heute nicht mehr existierenden Männerhaus benannt wird. Die Objekte Hüllblatt, Backschale und Herdschale stehen in einer besonderen Verbindung zur Frauenseite. Hierzu wurde auch gesagt, daß sich die Namen der Herdschale auf eine weibliche Ahnenfigur beziehen, die an dieser Stelle bestattet wurde. Das Hüllblatt, welches in sehr verschiedener Weise verwendet werden kann, ist hier gedacht als Sitzunterlage der Frauen. Die Herdschale ist ebenfalls wesentlicher Bezugspunkt der Frau, da in ihr auf der Sagobackschale die tägliche Nahrung von den Frauen zubereitet wird. 
Die Erwähnung der Schlange bezieht sich auf die Kampfkraft des Dorfes. Diese Schlange zieht wie eine Reihe von Kriegern gegen das Feinddorf, sie umzingelt wie diese Krieger den Feind. Nach dem Kampf kehrt sie jedoch in das Dorf zurück, und es müssen dann Schweine und Hühner getötet werden, da sich sonst diese Schlange gegen die eigenen Dorfbewohner richtet und ihnen Verderben bringt.

In einer Mythe über die Gründung des Totenlandes wird auch darauf hingewiesen, daß das Totenland wie eine neue Siedlung eingerichtet wird; es werden Rotang, Herdschale und Pandanus-Stelzen aufgezählt. Die Pandanus-Stelzen sind die Pfosten, mit denen der Ort gleichsam verankert wird. Ferner wird davon gesprochen, daß Erde vom alten Ort mitgebracht und daß Gras angepflanzt wird.

Als besondere Handlungen bei einer Siedlungsverlegung erwähnte man, daß bestimmte Gräser, Krötenarten und Erde vom westlichen, mittleren und östlichen Dorfteil mitgenommen würden. Wenn diese Dinge an eine andere Stelle im Wald gebracht worden sind, so soll in der alten Siedlung keine "Kraft» mehr vorhanden sein, unter den Dorfbewohnern entstehen Streit und Uneinigkeiten. Schließlich ziehen die Leute vom Dorf weg und gehen in die neue Siedlung. Von den ersten Ansiedlern wird eine Kokospalme gepflanzt. Man sagt, daß einer der ersten Ansiedler stirbt, sobald diese Kokospalme die ersten Früchte getragen hat. Diese Palme würde dann auf den ersten Ansiedler treten. Er wird zum mythischen Gründer des Dorfes. Sein Leichnam soll in Stücke zerlegt werden. Sein Haupt wird im westlichen Dorfteil (ambuwe, KrokodilKopf), sein Unterkiefer im östlichen Dorfteil (nganiwe, Krokodil-Schwanz) bestattet, die übrigen Körperteile hingegen legt man im mittleren Dorfteil (indeləpma) nieder. Diese Dorfrichtung entspricht der Entwässerung, ambuwe liegt stromaufwärts, nganiwe liegt stromabwärts. Nicht nur die heutige Siedlung wie Gaikorobi hat diese Ausrichtung, sondern auch frühere Siedlungen wie Ngətəpmə sollen diese Orientierung gehabt haben.

In Gesängen (saikundi) berichtet man ebenfalls von Siedlungsgründungen. Im folgenden möchte ich eine solche Beschreibung kurz zusammenfassen. Aus dem Gesang erfahren wir, daß eine weibliche Ahnengestalt eine Siedlung gründet. Sie geht in den Wald hinein und sucht eine Stelle für eine Siedlung aus, bricht kleine Äste ab und markiert damit den Grundriß der Siedlung. Danach sammelt sie Palmblätter und verteilt sie auf dem Erdboden. Sie zündet ein Feuer an, beim Feuerreiben tränen ihre Augen im Rauch. Mit einem Bündel von Kokospalmblättern nimmt sie von dem Feuer und zerstreut es. Das Feuer lodert empor und beleuchtet die Siedlungsstelle. Ein Wind erhebt sich. Durch das Feuer wird der Ort in ein rotes Licht getaucht. Adler horchen auf das Krachen des Feuers und schlagen mit ihren Flügeln. Sie kreisen in der Luft, und ihre Flügel schlagen auf und ab. Sie nehmen Asche von dem Feuer und verstreuen sie. Die
Frau kehrt mit Palmblattstielen die Asche zusammen. Der Ort sieht schön aus, und die Frau freut sich. Sie sät Pflanzen aus, später bricht sie von den ausgesäten Gemüsepflanzen, um sie zu essen.

\section{Der Kultplatz}

Der Dorfgrundriß wird bei den Sawos, deren Dörfer nicht wie diejenigen der Iatmul auf Uferdämmen des Sepiks oder an Altwasser liegen und daher von der Topographie mitbestimmt werden, durch die langgestreckten Tanzplätze beherrscht. An den Seiten dieser Plätze ziehen sich die Längshügel hin, die meistens von Kokospalmen und Ziersträuchern bewachsen sind. An den Schmalseiten der Plätze stehen die Männerhäuser.

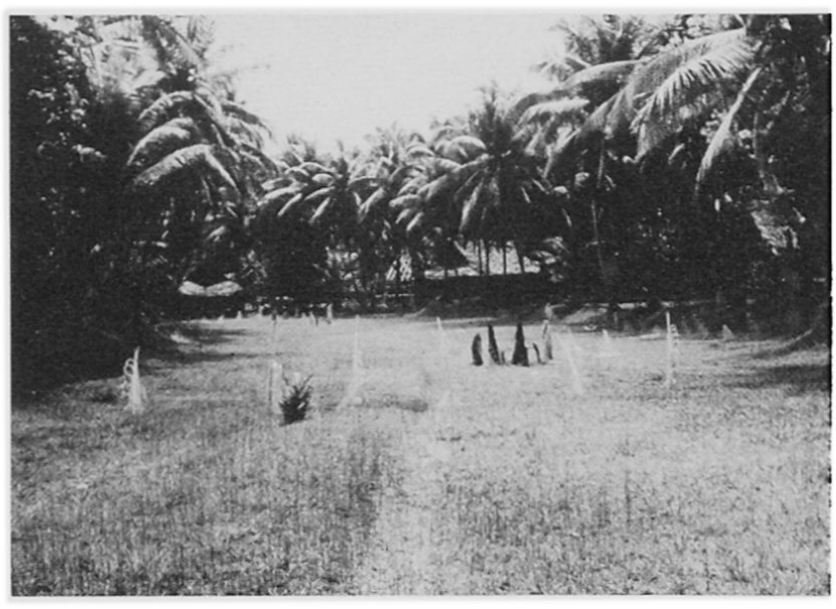

Abb. 1 Tanz- und Kultplatz Kosimbi von Gaikorobi (Sawos), mit Kultsteinen und weißen Palmblättern in der Mitte; links im Hintergrund das Männerhaus, auf den seitlichen Längshügeln Kokospalmen.

Dieser Platz ist den initiierten männlichen Mitgliedern der Gesellschaft vorbehalten. Früher wurde er nur während der Feste benutzt. Die alltäglichen Wege führen an den Seiten des Platzes vorbei. In der mythischen Vorstellungswelt werden diese Plätze mit Bachläufen gleichgesetzt, die Längshügel der Plätze bilden die Uferdämme. Andererseits spricht man bei Bächen von einem ihnen zugehörigen Tanzplatz, an dessen Ende Männerhäuser stehen.

Dieser Gedanke des «Im-Wasser-Lebens» kann sogar auf das ganze Dorf übertragen werden. Man spricht dann davon, daß die Leute eines Dorfes in einem djangu leben, einer Vertiefung in einem Bachbett. Diese Angleichung des Dorfes bzw. Kultplatzes an einen Wasserlauf zeigt sich besonders in der ideellen räumlichen Ausrichtung des Dorfes. Wie schon gesagt wurde, nennt man den westlichen Teil des Dorfes den Kopf des Krokodiles, den östlichen Dorfteil den Schwanz des Krokodiles. Ein Dorfgrundriß sollte so ausgerichtet sein, daß der Kopf des Krokodiles zum Oberlauf (Westen oder Norden), der Schwanz des Krokodiles zum Unterlauf (Osten oder 
Süden) blickt. Auch die Männerhäuser, die eine Vorder- und eine Rückseite haben, sollten nach diesem Orientierungssystem (Oberlauf/Unterlauf) gerichtet werden, indem die Vorderseite zum Oberlauf blickt. Die Befestigungstore der Siedlung von Gaikorobi waren früher ebenfalls nach dieser West-/OstRichtung orientiert, indem das erste Tor am westlichen Eingang des Dorfes stand, das dritte Tor hingegen den östlichen Teil des Dorfes schützte, in welchem die für das Dorf wichtigsten sakralen Gegenstände auch heute noch aufbewahrt werden.

Den Kultplatz nennt man auch tangək, wie den Fußboden eines Hauses, der aus einer Palmenrinde besteht, die auseinandergerollt werden muß. Besonders befähigte Männer können diesen Erdboden «verschließen»: Sie nehmen ein mattenähnliches Objekt, auf das sie einen Zauberspruch sagen, rollen es zusammen und legen es unter einen Stein oder eine Schlitztrommel. Diese magische Handlung soll bewirken, daß die Leute des Dorfes auf Kriegszügen nicht mehr erfolgreich sind, nicht mehr Jagdwild und andere Nahrung finden können. Das Auseinanderrollen und Öffnen dieser Palmenrinde symbolisiert hingegen Neubeginn und Fruchtbarkeit.

\section{Dorf und Frau}

In den bisherigen Angaben wurde hauptsächlich auf den Vergleich zwischen Dorf und Haus und auf die Ausrichtung nach einem Wasserlauf hingewiesen. Wir wollen im folgenden auf einen zusätzlichen Aspekt des Dorfes als Einheit eingehen. Früher schnitt man das Gras auf den Tanzplätzen nur zur Vorbereitung eines Festes. Die übrige Zeit ließ man das Gras wachsen, die Flächen zwischen den Wohnhäusern waren ebenfalls dicht zugewachsen. Über dieses Schneiden des Grases auf dem Tanzplatz sagte man, daß man die Frau des Dorfes säubere.

In einer Namenreihe (satkundi), die sich auf das Dorf bezieht, kommt ebenfalls zum Ausdruck, daß das Dorf mit einer Frau in Verbindung gesehen wird. Es sind Namen der Erde, d. h. der zuerst aus dem Wasser aufgetauchten Ur-Erde, die hier als Namen für das Dorf stehen. Weitere Namen beziehen sich auf den Muschelschmuck dieser Frau, der ihr um den Hals, an den Ohren usw. hängt. Dieser Schmuck schlägt aneinander und ertönt, wenn die Frau sich bewegt, sich von der einen auf die andere Seite dreht. Die Namen sagen ferner aus, daß die Männer die Frauen mögen, die sich für ein Fest schmücken, sie lieben ihr geschmücktes Dorf und ihre geschmückten Frauen. Hier wird deutlich, daß die Verbindung zwischen Dorf und Frau über den Erdboden hergestellt wird, die Erde, auf dem das Dorf steht. Dadurch wird auch verständlich, warum das Schneiden des Grases vom Tanzplatz als Säubern der Frau des Dorfes bezeichnet wird.

Einmal nahm ich mit einem älteren Mann von Gaikorobi an einem Initiationsfest in einem Nachbar- dorf teil. Wir verbrachten gemeinsam die ganze Nacht im Männerhaus und versuchten, wach zu bleiben, um die im Morgengrauen stattfindende Zeremonie zu beobachten. Trotz der Feuerstellen in der Mitte des Männerhauses froren wir beide. Da sagte der Mann aus Gaikorobi, daß wir kalt hätten, weil wir in einem fremden Ort seien. Man empfindet eine besondere Wärme im eigenen Dorf.

Um das Folgende zu verstehen, sei kurz auf die unterschiedliche Sitzweise von Männern und Frauen hingewiesen. Während die Männer entweder auf hölzernen Hockern oder auf Plattformen sitzen, besteht die Sitzweise der Frauen darin, daß sie im Wohnhaus unmittelbar auf dem Palmenrindenboden sitzen oder ein Hüllblatt einer Palme als Sitzunterlage benutzen, wobei sie die Beine ausstrecken oder auch kreuzweise übereinanderschlagen. Während eines Festes sitzen Frauen auf Hüllblättern und schauen zu. Wenn sie jedoch weggehen und ihr Hüllblatt mitnehmen, so sagte man mir, frieren die Männer und möchten nicht mehr tanzen. Man sagt auch, daß die Leute im Dorf auf den Schenkeln einer Frau gehen, die mit gekreuzten Beinen sitzt.

In der einheimischen Sprache der Sawos wird eine Gleichsetzung der Wörter für Siedlung und Kokospalme vollzogen. In der Bezeichnung für die Mitte der Siedlung Gaikorobi, Indeləpma, können wir den Bestandteil lopma (= topma, Kokospalme) erkennen oder an folgendem Namenpaar in der Namenreihe für das Dorf: Nge-sanglombi (nge, Haus, Dorf) und Təpma-sanglombi. Die Dorfgründer nennt man daher auch tapmanamba, die Kokospalmen-Leute. Die Kokospalme wurde immer wieder als Verkörperung eines weiblichen Ahnenwesens erklärt, ebenso wie die Arecapalme. In Zaubersprüchen wird in entsprechender Weise eine weibliche Ahnenfigur angesprochen. Wenn z. B. im Dorf keine Betelfrüchte mehr vorhanden sind, so sagt man, daß die Betel-Frau ihren Faserrock zugebunden hat, daß sie kein Menstruationsblut mehr fließen läßt, daß sie das Hüllblatt, auf dem sie sitzt, zusammengerollt hat.

In verlassenen Siedlungsstellen kann man häufig in dem wieder hochgewachsenen Busch Kokospalmen finden. Neben anderen Merkmalen, wie Kulthügeln und Kultsteinen, sind sie ein deutliches Zeichen für eine frühere Besiedlung, da Kokospalmen nicht in den Gärten angepflanzt werden. Die Einheimischen sagen, daß die Kokospalmen langsam zugrundegehen, wenn die Leute eine Siedlung verlassen. Die Kokospalmen sollen das Sprechen der Menschen hören können. Dort, wo sie den Rauch von Feuerstellen riechen, können sie bleiben und gut wachsen. Daher könnten sie auch nicht in den Gärten hochwachsen und Früchte tragen.

\section{Rituelle Handlungen für das Dorf}

Rituelle Handlungen in Beziehung zum Dorf haben als Ziel, die Dorfgemeinschaft zu stärken und Krisen, 
die dem Dorf drohen, abzuwenden. In einer solchen Handlung, die als "Aufrichten des Dorfes» bezeichnet wird, schlagen Vertreter verschiedener Klane mit der Blattrippe einer Kokospalme auf den Erdboden. Sie beginnen in jenem Dorfteil, der Krokodil-Kopf genannt wird, und gehen dann zum östlichen Dorfende, zum Krokodil-Schwanz. Das Schlagen mit Blattrippen der Kokospalme auf den Erdboden konnte auch in anderen rituellen Anlässen beobachtet werden; ich möchte es als ein plötzliches Vergegenwärtigen der vergangenen Ahnenwelt interpretieren, denn diese Ahnen sind es, die dem Dorf Unterstützung geben müssen.

Auf dem Kultplatz befinden sich vor den Männerhäusern Steinsetzungen. Diese ca. $1 \mathrm{~m}$ hohen Steine stehen in Verbindung mit der Kriegführung und mit der Kampfstärke des Dorfes. Umgestürzte Steine zeigen eine Schwäche in der Verteidigungsbereitschaft des Dorfes an. Wenn ein Stein umgefallen ist, so sagt man, daß ein Ahne umgefallen sei. Früher konnte dann kein Krieg gewonnen werden, und heute, nach dem Verbot der traditionellen Kopfjagd durch die ehemalige Kolonialverwaltung, hat man dann keinen Erfolg in der wirtschaftlichen Entwicklung des Dorfes. Früher wurde in das Loch des wieder aufzurichtenden Steines der Schädel eines Getöteten hineingelegt. Mit Hilfe eines Knochen-Orakels wurden jene bestimmt, die während der Nacht den Stein aufrichteten. Ihre Identität, ihre Namen, sollten geheim bleiben, besonders gegenüber traditionell feindlich eingestellten Dörfern.

Im November 1972 konnte ich Teile des Festes zur Aufrichtung eines Steines beobachten. Während der Nacht blies man auf den langen Bambusflöten. Im ganzen Dorf wurden junge Palmblätter (kinsan) in den Boden gesteckt (vgl. Abb. 1), an Stellen, an denen sich die Vorfahren befinden. Mit diesen Palmblättern wurde auch auf den Tanzplatz geschlagen. In der gleichen Nacht führten zwei Altersgruppen einen symbolischen Kampf aus. Mit Rotangseilen, die auch als Schlange bezeichnet werden, wurden die Männer der jüngeren Altersgruppe eingekreist, die dann mit Hilfe von kreisförmig in den Boden gerammten langen Holzstöcken (ndəme) aus dieser Umzingelung auszubrechen versuchten. In diesem Rotang bzw. dieser Schlange können wir einen Ausdruck für die Kampfkraft des Dorfes sehen. Auf einen Kriegszug nahmen früher die Anführer (suvundimi) diese Schlange mit, um den Feind einzukreisen. Diese Schlange ruht im ältesten Kult- und Tanzplatz von Gaikorobi.

\section{Die Kwanga}

Die Kwanga (3) leben in Nordost-Neuguinea, südlich des Torricelli-Gebirges, das parallel zur Nordküste von Neuguinea verläuft. Sie bewohnen ein Gebiet von ungefähr $650 \mathrm{~km}^{2}$, ihre Bevölkerungszahl beträgt etwa
12000 Menschen. Die Kwanga legen ihre Siedlungen auf den sehr schmalen Kämmen der meistens in nordsüdlicher Richtung verlaufenden Hügelketten an, auf einer Höhe zwischen 100 und $200 \mathrm{~m}$. Die Dörfer sind umgeben von niedrigem Sekundär-Regenwald und Gartenanlagen, erst in einer größeren Entfernung vom Dorf trifft man auf dichteren und höheren Regenwald. Die Einwohnerzahl der Kwanga-Dörfer beträgt durchschnittlich 330 Bewohner und variiert zwischen ca. 100 und 800 Menschen pro Dorf. Die Siedlungen weisen eine starke Streuung auf und bestehen aus locker zusammengefügten Weilergruppen. Die Dörfer besitzen keinen zentralen Punkt; anders als bei den Abelam oder Iatmul und Sawos finden wir bei ihnen keine hoch aufragenden Kultbauten oder Kultplätze, die das Bild des Weilers oder Dorfes dominieren. Die Kwanga betreiben Brandrodungsfeldbau, ihre Hauptanbaupflanze ist der Yams. Jagd und Sammeltätigkeit sind von untergeordneter Bedeutung; anhand der zentralen Stellung, die die Jagd im rituellen Bereich innehat, läßt sich vermuten, daß sie früher wichtiger war. Der Kontakt der Kwanga mit der europäischen Zivilisation begann im größeren Umfang nach dem Zweiten Weltkrieg. Ende der 60er Jahre pflanzte man die ersten Kaffeebäume, die heute eine wichtige Einnahmequelle bilden.

\section{Die kosmologische Einordnung}

Bei den Kwanga finden wir ähnlich wie bei den Sawos eine Orientierung nach vier Hauptrichtungen vor: Kerlaka Minimbia (Osten), Kelemontlo Apmanumi (Westen), Krumbawa Telewakri (Norden), Singiadenge Arkodenge (Süden). Mit den gleichen Namen werden auch die dort lebenden Menschen bezeichnet. Diese Namen sind jedoch das Wissen von nur wenigen Männern, im alltäglichen Sprachgebrauch finden wir die häufigere Orientierung nach Oberlauf und Unterlauf bzw. oben/unten vor. Nach diesem Raummuster werden die einzelnen Weiler eines Dorfes oder auch nebeneinanderliegende Dörfer zueinander in Beziehung gesetzt. So spricht man von dem «oben liegenden Dorf», von den "Leuten flußabwärts» usw.

In den Mythen über den Ursprung des Kosmos und der Menschen stoßen wir bei den Kwanga auf Überlieferungen von einem Ur-Stein. Dazu soll folgende Aussage des Mannes Apkolasa vom Dorf Kuyor wiedergegeben werden: "Wir sind aus einem Stein entstanden. Eine Schlange lag auf dem Stein, den man Waimula nennt. Dieses Dorf gab es noch nicht, Menschen, Schweine und Käfer gab es noch nicht. Wasser bedeckte alles. Der Stein lag in diesem Wasser, und eine kleine Schlange ruhte auf dem Stein. Der Stein wurde schwanger und hatte einen sehr dicken Bauch. Er wollte ein Kind gebären und schrie. Ein Mann hörte davon, fürchtete sich und rannte weg. $\mathrm{Er}$ erzählte einem anderen davon, der nachschaute: "Ah, eine Frau!" Er band den Stein an einen Stock und trug 
ihn in das Haus. Im Haus gebärte der Stein ein Kind und knallte. Das Kind kam hervor und schrie. Der Name des Kindes ist Wolokayi. Das Wasser war zurückgegangen. Nebel hatte alles eingehüllt, es war dunkel gewesen. Als der Stein auseinanderbrach, stiegen der Nebel und die Wolken empor. Der Mann Wolokayi Kerengwa entstand. Von ihm stammen wir Menschen ab.»

Aus dem Schädel von Wolokayi entstand, einer Kwanga-Mythe folgend, die Kokospalme. In einem Dorf der Kwanga wird heute noch ein Stein aufbewahrt, der sich auf diese mythische Überlieferung bezieht. Aus diesem Ur-Stein, der manchmal auch als Ei bezeichnet wird, entstanden die Schweine und die Menschen. In kultischen Gesängen werden daher auch Schweine Stein-Kinder genannt. "Aufstellen von Steinen» ist ein bildlicher Ausdruck für die Ausübung des Kultes, der der Fülle von Jagdwild und Gartenpflanzen dient. Diese Steine werden auf dem Kultplatz für Feuerstellen aufgebaut, an denen man die Nahrung für die in den Kult Einzuweihenden zubereitet.

\section{Die Dorfgründung}

Im folgenden beziehe ich mich auf den Ausschnitt eines Gesanges, in dem eine kriegerische Auseinandersetzung zwischen zwei Siedlungen der Kwanga beschrieben wird. Die Leute der einen Siedlung werden durch die Kriegsereignisse vertrieben und ziehen fort, sie lassen sich an einem anderen Ort nieder. Die neue Ansiedlung wird damit umschrieben, daß die Leute Kokospalmen und Brotfruchtbäume pflanzen und die alles überwuchernden Schlingpflanzen entfernen. Später wachsen Bananenpflanzen und Bambus hoch. Die Leute ziehen Schweine und Hunde auf, sie entfachen ein Feuer, und Rauch zieht empor. Sie legen Steine hin und bauen eine Feuerstelle, womit angedeutet wird, daß sie mit den Vorbereitungen für den Kult beginnen.

\section{Der Kultplatz}

Bei den Kwanga gibt es zwei Arten von Tanz- und Kultplätzen. Während der Tanzplatz, der mit dem sukutja-Fest verknüpft ist, im Mittelpunkt des Dorfes liegt und durch auf dem Boden liegende Holzstangen markiert wird, befindet sich der Tanzplatz des $k w a-$ ramba-Kultes am Rande des Dorfes im verborgenen. Hier soll allein auf letzteren näher eingegangen werden. Vom Dorf aus führt ein schmaler Weg, der nur den Kult-Mitgliedern zugänglich ist, auf den kreisförmigen Platz; er ist vom Dorf aus nicht zu sehen. An seinen Seiten stehen einzelne Häuser, die den in den Kult eingeführten Männern als Schlafhäuser dienen können. Für die kwaramba-Zeremonien baut man an einer Seite des Platzes zusätzlich ein größeres Kulthaus (kwaramba aka), das nach den
Zeremonien wieder verfällt. Auch andere Objekte, die mit dem Kult zusammenhängen, werden nicht für eine Dauer, als permanente äußere Erscheinung einer jenseitigen Wesenheit, angelegt. Das ist der Grund, warum man in Kwanga-Dörfern meistens keine kwaramba-Kulthäuser antrifft. Der Tanzplatz (kondome) wird während des Kultes zeitweise durch einen zusätzlichen Zaun abgegrenzt. Vor den Festen wird sämtliches Gras auf dem Platz entfernt. Die Sitzplätze für die Männer befinden sich auf den Seiten des Platzes.

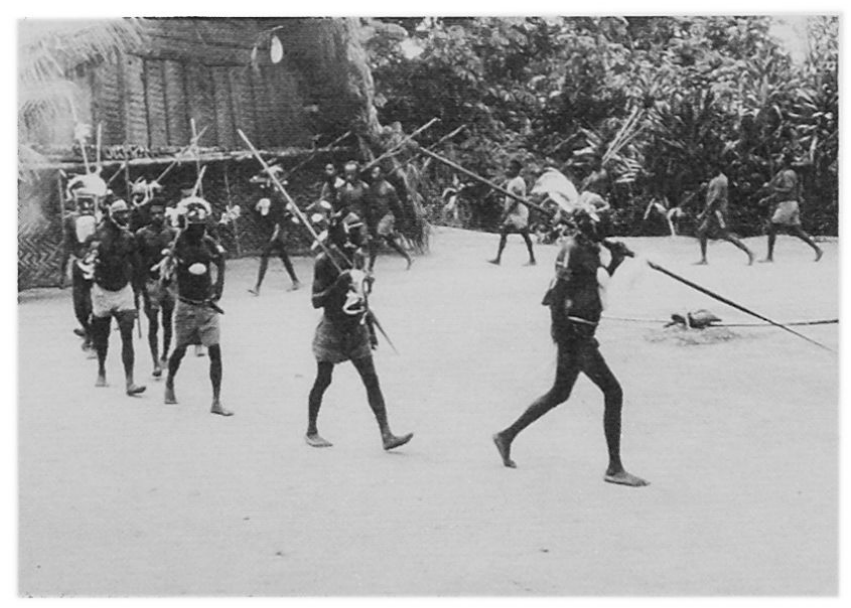

Abb. 2 Kultplatz in Mamsi (Bongos-Gebiet, Kwanga); Jäger tanzen um ein getötetes Tier (Wallaby), links im Hintergrund die Fassade des Kulthauses.

Besonders an den Verhaltensvorschriften können wir erkennen, wie diesem Platz eine besondere Stellung zugewiesen wird. So ist es z. B. nicht erlaubt, auf dem Platz zu rauchen, da man sonst krank werden würde. Ebenso legen die Männer ihre Netztaschen ab, bevor sie zum Tanz auf den runden Platz eilen. Der kwaramba-Kult ist eng verbunden mit der Jagd auf Wildschweine. Männer aus Nachbardörfern jagen für den Kult Wildschweine und bringen sie auf den Tanzplatz. Bevor sie den Platz betreten, schlägt ihr Anführer mit einem Stock oder einem zusammengerollten Hüllblatt einer Palmblüte auf den Boden. Das getötete Jagdwild legt man in die Mitte des Platzes, um das Tier herum tanzen die Jäger (vgl. Abb. 2). Nach einer Weile bleiben sie in einem Halbkreis stehen, nehmen einen Pflanzenstengel in den Mund und spucken dessen Saft aus. Auf diese Weise rituell gereinigt, eilen sie vom Platz hinweg. In der Mitte des Platzes hat man zu Beginn eine Grube ausgehoben, in die bestimmte Substanzen (z. B. Knochen) hineingelegt werden, die als Jagdzauber dienen sollen. Neben oder auf diese dann wieder zugeschüttete Grube werden später die gejagten Tiere gelegt.

Die angeführte isolierte Lage dieser Kultplätze im Dorf drückt die starke Geheimhaltung aus, die diese 
Plätze umgibt. Die betonte Geheimhaltung des Kultes führt dahin, daß bestimmte Handlungen außerhalb des Dorfes im Wald ausgeführt werden. Man bezieht das Waldgebiet außerhalb des Dorfes in den Kultbereich mit ein.

\section{Kokospalme und Brotfruchtbaum}

Die Kwanga-Dörfer bilden nicht in sich geschlossene selbständige Einheiten wie die Dörfer am Mittelsepik; dies wird an der Siedlungsweise deutlich mit den teilweise weit voneinander entfernten Weilern. In einzelnen Fällen können die äußersten Weiler eines Dorfes bis zu einer Stunde Wegzeit voneinander entfernt liegen. Während am Mittelsepik die Dorfgemeinschaft fast endogam ist, gibt es zwischen den Kwanga-Dörfern zahlreiche Heiratsverbindungen. Das Gefühl der Zugehörigkeit richtet sich stärker nach dem eigenen Weiler und weniger dem Dorf zu. Auf die Frage nach dem Wohnort erhält man deshalb häufig einen Weilernamen. Auch vom äußeren Erscheinungsbild her läßt sich kaum feststellen, wo die Dorfgrenzen liegen.

In Gesprächen mit Einheimischen bemerkt man, worin sie Kriterien für die Dorfgemeinschaft sehen. $\mathrm{Da}$ ist das Netz der Heiratsbindungen und Tauschbeziehungen, das innerhalb eines Dorfes immer noch enger und größer ist als das zwischen verschiedenen Dörfern. Ferner ist die gemeinsame Ausübung des Kultes wichtig. Wenn die Teile eines Dorfes zu sehr auseinanderwachsen, die Wege zu lang werden, tendiert man zur Dorfspaltung, mit getrennt ausgeführten Kultfesten.

Im ersten Beispiel, vom Mittelsepik, sollte gezeigt werden, wie die Bedeutung des Dorfes für die gegenwärtig darin lebenden Menschen stark von den im Dorfboden begrabenen Ahnen abhängig ist; von diesen Ahnen bzw. den im Dorfboden ruhenden Ahnenknochen wird die Verbindung zur Gegenwart gezogen. Die Abstammung sieht man primär über die väterliche Linie, symbolisch ausgedrückt durch die Knochensubstanz, die jedermann von der väterlichen Seite erhält. Die Kwanga hingegen drücken ihre Zugehörigkeit zu einem Ort mit dem Wort «Blut» aus, man ist «Blut» eines bestimmten Ortes. Die Blutsubstanzen von Mann und Frau bilden das entscheidende Element für einen neuen Menschen.

In rituellen Gesangstexten mit einer sehr bildhaften Sondersprache, die nur wenigen Männern verständlich ist, nennen die Kwanga folgende Bezeichnungen anstelle von Dorf: Kokospalme, Brotfruchtbaum, Feuer oder glimmendes Holzstück, duftendes Blatt, Taro, Bambus. Die Termini für Kokospalme und Brotfruchtbaum, die paarweise auftreten, nennt man jeweils an erster Stelle.

Im Landschaftsbild sind auch auf weit entfernten Hügeln Dörfer daran zu erkennen, daß sich die Palmkronen der Kokospalmen von der übrigen
Vegetation abheben, ebenso wie die hoch aufragenden Brotfruchtbäume. So wird in einem Gesang beschrieben, wie ein Mann verschiedene Dörfer besucht und an Festen teilnimmt und erst nach längerer Zeit den Heimweg antritt; er erblickt die Kokospalmen und Brotfruchtbäume seines Dorfes und kehrt zurück.

Die Kokospalme bietet eine wesentliche Zusatznahrung im Dorf. Wie am Mittelsepik, steht auch bei den Kwanga die Kokospalme mehr als andere Fruchtbäume in einer speziellen Beziehung zu den Dorfbewohnern. Nach einem Todesfall vollziehen die Trauernden ein gemeinsames Mahl, die Kokosnüsse des Verstorbenen werden von den Palmen heruntergeworfen, alle sollen davon essen. Auf diese Weise möchte man denjenigen, der durch magische Handlungen den Tod eines Dorfmitgliedes herbeigeführt hat, entdekken, da nach dem Verzehren der Kokosnuß der Totengeist des Verstorbenen diesen aufsucht und verfolgt.

Der Brotfruchtbaum, häufig im Dorf angepflanzt, bietet den Dorfbewohnern ebenfalls eine wichtige Nahrung mit seinen Früchten. Seine Blätter dienen zum Einwickeln von Nahrung, als Sitzunterlage usw. Im folgenden sei auf eine Verwendung hingewiesen, die den Brotfruchtbaum in eine Beziehung zur gebärenden Frau stellt. Der Mann einer schwangeren Frau nimmt ein Blatt des Brotfruchtbaumes und tropft auf dieses Blatt Blut von seinem Penis. Er staut einen kleinen Bachlauf, läßt in das Wasser ebenfalls PenisBlut tropfen, durchbricht den Damm, und das Blut fließt mit dem Wasser ab. Mit dem Blatt des Brotfruchtbaumes reibt eine Frau auf dem Bauch der Schwangeren von oben nach unten. Danach hängt der Mann das Blatt an einen Baumzweig, wo es vom Winde hin- und herbewegt wird. Zu gleicher Zeit soll das Kind aus dem Leib der Schwangeren geboren werden. Die Nabelschnur des Kindes wickelt man in ein Brotfruchtbaum-Blatt ein und legt sie auf einen Baumzweig. Erst später wird sie von der Frau in einen Bach geworfen.

GELL (1975: 39) hat am Sepik-Oberlauf im Dorf Umeda festgestellt, daß die Kokospalme als ein Symbol der agnatischen Kontinuität gilt. In der gleichen Weise werden von den Umeda die Brotfruchtbäume mit einer menschlichen Siedlung assoziiert (GELL 1975: 285), jedoch mehr mit den Hausbauten in Gartenanlagen und damit dem Geburtsort der Umeda. GELL (1975: 289) zieht die Schlußfolgerung: "The breadfruit, in terms of tree-symbolism, stands on the side of regeneration, as opposed to the coconuts, standing for ancestral values.»

Das Beispiel der Kwanga legt eine ähnliche Interpretation nahe: Der Verstorbene wirkt durch seine Kokospalme über den Tod hinaus fort, während das Blatt von Brotfruchtbaum die Geburt eines Menschen fördert und als Hülle für seine Nabelschnur dient. Trotzdem sind beide, Kokospalme und Brotfruchtbaum, symbolische Ausdrücke für die menschliche Siedlung. 


\section{Rituelle Handlungen und Dörfer-Verband}

Im kwaramba-Kult können wir feststellen, wie verschiedene Dörfer einen Kult-Verband bilden, zur Ausübung des Kultes ist die Zusammenarbeit mehrerer Dörfer notwendig. Die gleichen Dörfer haben sich früher in kriegerischen Auseinandersetzungen gegenseitige Hilfe geleistet. Der kwaramba-Kult stellt durch die feste Abfolge von Kultfesten die einzelnen Dörfer in eine Reihenfolge und durch die dabei stattfindenden gegenseitigen Tauschhandlungen in ein Beziehungsnetz. Die in einer lockeren Siedlungsgemeinschaft stehenden Weiler werden in ein vielfältiges Netz von wirtschaftlichen und kultischen Verpflichtungen eingefügt.

\section{Schluß}

Die Untersuchung der gedanklichen Beziehungen der Sawos und Kwanga $\mathrm{zu}$ ihren Siedlungen hat uns gezeigt, wie die urzeitlichen Ereignisse, die in den Mythen überliefert werden, das Grundmuster für die heutigen Siedlungen geben. Neben der Aufgliederung in die vier Himmelsrichtungen ist es vor allem die Orientierung nach einem Flußlauf, die die räumliche Einordnung für die Siedlung bietet. Bei den Sawos haben wir gesehen, daß die Siedlung einem Haus gleichgesetzt wird, von besonderer Bedeutung sind die Toten, die am Anfang im Dorfboden bestattet werden. Bei den Kwanga wird die Verbindung zum eigenen Dorf mit dem Wort für Blut angegeben. Der Kultplatz

\section{Anmerkungen}

(1) Vgl. dazu Rapoport 1976 und darin besonders den Artikel von Lucy Jayne Kamau: "Conceptual Patterns in Yoruba Culture", S. 333-364.

(2) Von 1972-74 führte ich bei den Sawos im Dorf Gaikorobi ethnologische Untersuchungen aus; die Forschungen konnten stattfinden dank der finanziellen Unterstützung durch den Schweizerischen Nationalfonds zur Förderung der wissenschaftlichen Forschung; der Feldaufenthalt war Teil der SepikExpedition unter der Leitung von Prof. Dr. Meinhard Schuster, Ethnologisches Seminar der Universität Basel. Vgl. zur Kultur der Sawos Schindlbeck 1980.

(3) Von 1979-80 arbeitete ich mit Unterstützung der Deutschen Forschungsgemeinschaft bei den Kwanga im Gebiet von Bongos. Eine Darstellung ihrer Kultur ist in Vorbereitung.

Zeichnung und Fotos: M. Schindlbeck und die mit ihm assoziierten Kulturelemente sind bei den Sawos wichtig für die Kriegführung, bei den Kwanga für die Nahrungsfülle. Attribute der Frauen, wie Herdschale, Backschale, Hüllblatt, können bei den Sawos als Symbole für die Wärme und den Mittelpunkt der eigenen Siedlung gesehen werden. In beiden Kulturen gilt die Kokospalme als bildlicher Ausdruck für die Siedlung und die Abstammung von den Ahnen.

\section{Summary}

In this paper I analyse the concepts about settlement of two cultures in the Sepik region of Papua New Guinea. These concepts include ideas of space orientation. Further points of the analysis are: the founding of villages, settlement patterns, ceremonial ground, symbolic expressions for their villages and rituals for village strength and unity. Among the Sawos we have the comparison between village and house, the founding of a village is compared to the building of a house. The first settlers of a village who are called the "coconut people» are of great importance for the settlement. The ceremonial ground contains symbolically cultural elements which are the centre of the village. The Sawos speak of their village as a female being. The $\mathrm{K}$ wanga and the Sawos have as symbol for their village the coconut palm. Among the Kwanga the breadfruit-tree is associated with settlement and human birth.

\section{Literaturverzeichnis}

BÜHLER, A. (1960): Der Platz als bestimmender Faktor von Siedlungsformen in Ostindonesien und Melanesien. In: Regio Basiliensis 1, 2, S. 202-212.

GELL, A. (1975): Metamorphosis of the Cassowaries. Umeda Society, Language and Ritual, London.

RAPOPORT, A. (ed.) (1976): The Mutual Interaction of People and Their Built Environment. A Cross-Cultural Perspective, The Hague/Paris.

SCHINDLBECK, M. (1980): Sago bei den Sawos (Mittelsepik, Papua New Guinea). Untersuchungen über die Bedeutung von Sago in Wirtschaft, Sozialordnung und Religion. Basler Beiträge zur Ethnologie, 19, Basel.

SCHMITZ, C.A. (1955): Balam. Der Tanz- und Kultplatz in Melanesien als Versammlungsort und mimischer Schauplatz. Emsdetten (Westf.) 